This manuscript is a preprint and has been submitted for publication. Subsequent versions may have slightly different content. The DOI of the peer reviewed publication will be provided if accepted. Please contact the authors if you have any questions or comments on this manuscript. 


\section{Advancing floating macroplastic detection from space using hyperspectral imagery}

Paolo Tasseron ${ }^{1, *}$, Tim van Emmerik ${ }^{1}$, Joseph Peller ${ }^{2}$, Louise Schreyers ${ }^{1}$ and Lauren Biermann ${ }^{3}$

${ }^{1}$ Hydrology and Quantitative Water Management Group, Wageningen University and Research, 6708 PB

Wageningen, The Netherlands; tim.vanemmerik@wur.nl (T.v.E.); louise.schreyers@wur.nl (L.S.)

2Plant Sciences Group, Wageningen University and Research, 6708 PB Wageningen, The Netherlands;

joseph.peller@wur.nl (J.P.)

3Plymouth Marine Laboratory (PML), Earth Observation Science and Applications, Plymouth,

United Kingdom of Great Britain and Northern Ireland; lbi@pml.ac.uk (L.B.)

*Correspondence: paolo.tasseron@wur.nl

Abstract: Airborne and spaceborne remote sensing (RS) collecting hyperspectral imagery provides 11 unprecedented opportunities for the detection and monitoring of floating riverine and marine plastic debris. 11 However, a major challenge in the application of RS techniques is the lack of fundamental understanding of spectral signatures of water-borne plastic debris. Recent work has emphasised the case for open-access hyperspectral reflectance reference libraries of commonly used polymer items. In this paper, we present and analyse a high-resolution hyperspectral image databaseof a unique mix of 40 virgin macroplastic items and vegetation. Our double camera setup covered the visual to shortwave infrared (VIS-SWIR) range from 40012 13 14 15 16 $1700 \mathrm{~nm}$ in a dark room experiment with controlled illumination. The cameras scanned the samples floating in 17 water and captured high-resolution images in 336 spectral bands. Using these resulting reflectance spectra as a baseline, a linear discriminant analysis was done to determine which wavelengths are more useful for discriminating between water and mixed floating debris, and vegetation and plastics. We then examined current Sentinel-2 and Worldview-3 satellite techniques, and the Normalised Vegetation Difference Index (NDVI) and Floating Debris Index (FDI) to determine why they work, and how they could potentially be improved. These findings could be used to enhance existing efforts in monitoring macroplastic pollution, as well as form a baseline for the design of future multispectral RS systems.

Keywords: remote sensing, Sentinel-2, earth observation, plastic monitoring, spectral reflectance 
Plastic pollution in natural environments has adverse effects on wildlife, habitat, and human wellbeing. An estimated 19-23 million metric tonnes of plastics enter aquatic ecosystems annually, which is predicted to increase by an order of magnitude in the coming decades [1, 2]. Meijer et al. [3] estimated that 0.8-2.7 million metric tonnes of macroplastic enter the oceans through rivers on an annual basis. Such global model estimates depend on reliable observational data, which has led to many studies on different monitoring techniques to improve large-scale detection and quantification of riverine macroplastic loads and plastics accumulated on beaches, lakeshores, and riverbanks. Crows-based observations can be used to estimate riverbank macrolitter abundance over large areas [4,5]. More localised macroplastic assessments used floating booms [6], bulk water samples [7], and visual counting from bridges, riverbanks and quay walls [8, 9]. Despite these increasing efforts, data collection is inconsistent over time and space in riverine and coastal environments [10, 11]. Furthermore, the current methods to quantify macroplastic abundance are often labour-intensive, costly, require additional or specialist equipment, and are geographically limited to local scales [5, 12].

Recently, remote sensing (RS) collecting multi- to hyperspectral imagery has started to show far-reaching potential for detection and monitoring of riverine and marine plastic pollution [13, 14]. For example, Topouzelis et al. [15] and Themistocleous et al. [16] successfully identified large artificial plastic targets in coastal zones using Sentinel-2 imagery. Biermann et al. [17], Kikaki et al. [18], and Garaba et al. [19] categorised floating litter in marine environments based on varieties in spectral reflectance of different materials. On a smaller laboratory scale, Goddijn-Murphy and Dufaur [20] showed that the reflectance of plastic targets floating on water surfaces roughly corresponded with the reflectance of similar materials in a controlled laboratory setting. However, this relation is extremely sensitive to the type of plastic, transparency, shape, and surface characteristics [20]. In fact, Martínez-Vicente et al. [21] emphasised it is a challenge to confirm whether reflection characteristics observed in the laboratory can be used for detecting plastic litter in natural water systems. Additionally, natural and human-made items each have unique optically active spectral reflectance signatures, hampering the detection of mixed floating litter aggregates.

Several fundamental experiments exploring spectral signatures of virgin and harvested plastics have now been 53 conducted in controlled environments [22-25]. These studies encourage the establishment of ultraviolet (UV) to 54 shortwave-infrared (SWIR) hyperspectral reflectance libraries of plastic items. Garaba and Dierssen [13] presented a large spectral reflectance dataset of wet and dry marine-harvested, washed-ashore, and virgin macroplastics in the UV-SWIR range using a hyperspectral spectroradiometer. The complementary study of 55 56 Knaeps et al. [23] extended existing datasets by adding spectral measurements of floating and submerged plastics with varying levels of water turbidity. Another indoor experiment by Corbari et al. [26] focussed on spectrally characterising common plastic polymer types to determine the uniqueness of their spectral signatures 58 59 in the UV-SWIR range. While the methods of these studies differed, the results were similar and have 60 consistently shown that spectral shapes of plastic items consist of peak reflection and absorption features unique to the material composition [13]. The same has been seen in spectrograph imaged beach-harvested 62 plastics measured in the 1000-2500 nm SWIR range [27]. Indeed, based on the knowledge that plastics have 63 unique spectral signatures, near-infrared (NIR) spectroscopy is already operationally used in the sorting of 
plastics in recycling plants [16]. Despite these fundamental studies, the potential of hyperspectral reflectance 66 libraries for macroplastic detection from space is largely unexplored.

Outside of laboratory settings, multispectral data acquired by airborne or spaceborne remote sensors have been 68 used for detection of floating debris [15-17, 19, 21, 22, 28]. As with lab-based studies, detection algorithms here 69 leveraged absorption features, or spectral signatures, and used different combinations of RS bands to create feature extraction indices. For example, Rokni et al. [29] proposed various feature extraction indices for floating debris detection, which were complemented and tested by Themistocleous et al. [16] using multispectral data from the Sentinel-2 satellites and an Unmanned Aerial Vehicle (UAV). Also using Sentinel-2 data, Biermann et al. (2020) used a novel Floating Debris Index (FDI) for subpixel detection of floating debris and was able to discriminate plastics from natural materials using their spectral signatures. As opposed to hyperspectral imaging systems, these studies are limited by a given number of bands representing central wavelengths, often with a 20-40 nm range around the central bandwidth.

While rigorous lab-based studies have provided thorough insights into hyperspectral

polymer-specific diagnostic absorption features, there has been little reproducibility in natural systems. In an effort to improve the fundamental basis of reflectance-based plastic identification, we present a high-resolution hyperspectral image dataset of a unique mix of plastics and vegetation. We conducted hyperspectral measurements in the visual (VIS, $400 \mathrm{~nm}$ ) to shortwave infrared (SWIR, $1700 \mathrm{~nm}$ ) range of the electromagnetic spectrum. Our objectives were threefold, namely to (i) establish a high-resolution library of spectral signatures of virgin macro plastics, (ii) identify which wavelengths are most efficient in discriminating between plastics, vegetation, and water, and (iii) validate current satellite remote sensing techniques based on wavelengths measured with the Sentinel-2 and Worldview satellites. Our findings support existing efforts in monitoring macroplastic pollution using RS techniques and offer key information for the design of future airborne and spaceborne multispectral RS systems.

\section{Materials and Methods}

\subsection{Plastic samples description}

In this study, virgin plastic household items were collected from general household sources. A categorised overview of these plastics is shown in Figure 1. Out of 60.000 different types of plastic polymers, the most produced plastics by mass are low-density polyethylene (LDPE), high-density polyethylene (HDPE), polystyrene (PS), polyvinyl chloride (PVC), polypropylene (PP) and polyethylene terephthalate (PET) [30, 31]. These types 92 93 of plastic are the most abundant in freshwater systems and rivers, even though the distribution, types and 94 magnitude of plastic waste are variable [32-34]. PVC is rarely found floating due to its high density compared to water and is therefore not considered in this paper. Several examples of the remaining categories are 95 96 summarised in Table 1, as well as their specific gravity relative to water and common optical properties. The 97 distribution of polymer compositions is averaged over multiple freshwater systems, based on findings in Schwarz et al. [33] and Van Calcar and Van Emmerik [9] who demonstrated the macroplastic distribution can vary greatly for each environmental compartment. 
In addition to the virgin plastic collection, the database was complemented by riverbank-harvested macrolitter including plastics at different degradation states, and other anthropogenic debris. In total, $67 \%$ of the items were classified as plastics, divided into seven polymer categories. All information and statistics about the riverbankharvested macrolitter are found in the supplementary material of this article. The riverbank-harvested items
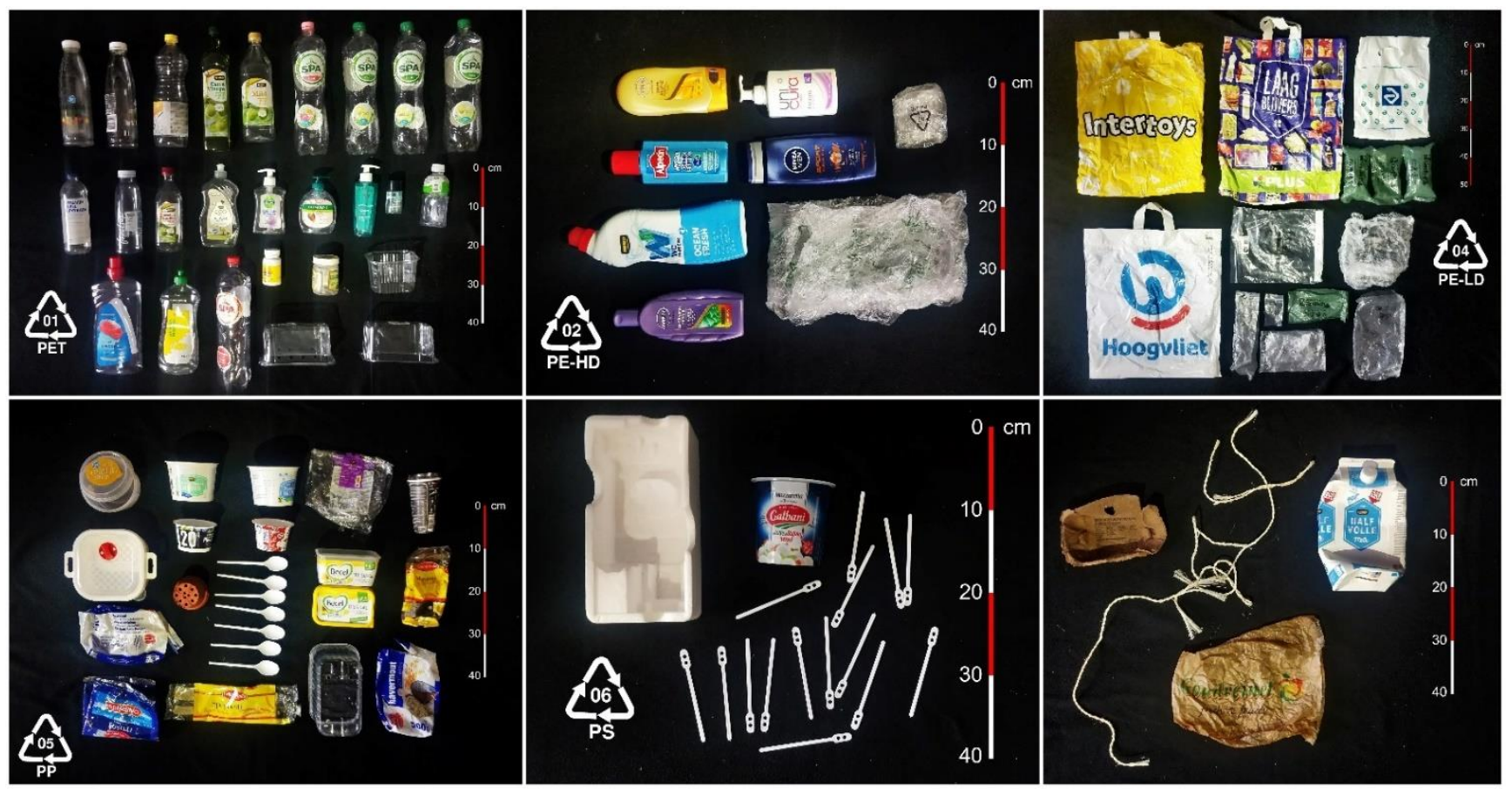

Figure 1. Virgin plastic collection subdivided into five polymer categories, and a non-plastic category

Table 1. Types of plastics in freshwater environments showing density, abundance, and examples of their use. [9, 20, 31, 33, 35]

\begin{tabular}{|c|c|c|c|c|}
\hline $\begin{array}{l}\text { Type of } \\
\text { plastic }\end{array}$ & $\begin{array}{l}\text { Specific } \\
\text { gravity } \\
\left(g * \mathrm{~cm}^{-3}\right)\end{array}$ & $\begin{array}{l}\text { Composition } \\
\text { distribution }\end{array}$ & $\begin{array}{l}\text { Optical } \\
\text { properties }\end{array}$ & Examples \\
\hline $\begin{array}{l}\text { low-density } \\
\text { polyethylene (LDPE) }\end{array}$ & $0.91-0.93$ & $17-42 \%$ & $\begin{array}{l}\text { (semi)transparent } \\
\text { clear/coloured }\end{array}$ & $\begin{array}{l}\text { cling film, garbage bags, } \\
\text { shopping bags }\end{array}$ \\
\hline $\begin{array}{l}\text { high-density } \\
\text { polyethylene (HDPE) }\end{array}$ & $0.94-0.96$ & $17-42 \%$ & $\begin{array}{l}\text { semi-transparent } \\
\text { white/coloured }\end{array}$ & $\begin{array}{l}\text { milk bottles, detergent bottles, } \\
\text { sandwich bags }\end{array}$ \\
\hline polystyrene (PS) & 1.04 & $11-17 \%$ & $\begin{array}{l}\text { opaque white, } \\
\text { grey specks }\end{array}$ & $\begin{array}{l}\text { plastic cutlery, food containers, } \\
\text { one-use cups }\end{array}$ \\
\hline polypropylene (PP) & $0.83-0.85$ & $11-30 \%$ & $\begin{array}{l}\text { semi-transparent } \\
\text { and coloured }\end{array}$ & $\begin{array}{l}\text { chip bags, drinking straws, } \\
\text { yoghurt containers }\end{array}$ \\
\hline $\begin{array}{l}\text { polyethylene } \\
\text { terephthalate (PET) }\end{array}$ & 1.37 & $<10 \%$ & Transparent/clear & $\begin{array}{l}\text { soft drink bottles, water bottles, } \\
\text { clamshell packages }\end{array}$ \\
\hline
\end{tabular}


In addition to the plastic samples described above, the spectral signatures of water and vegetation were also approximately 15 centimetres.

\subsection{Experimental setup}

The hyperspectral imaging of plastic items, water and vegetation was performed using a double camera setup. Together, the two cameras spanned the electromagnetic spectrum from 400 to $1700 \mathrm{~nm}$, covering the visual (VIS) to shortwave infrared (SWIR) range. The cameras were set up in a dark room, with controlled illumination.

Figure 2 gives a schematic overview of the system. The imaging in the VIS-NIR range was performed using the Specim FX10 camera (Konica Minolta Company, Oulu, Finland). It captures at a rate of $25 \mathrm{fps}$ in the VIS-NIR range of the spectrum (400-1000 nm) with a resolution of 1024 pixels per line scan, in 224 wavelength bands across the camera's spectral range. The effective pixel size is $20 \times 10 \mu \mathrm{m}$, and the spectral resolution is $5.5 \mathrm{~nm}$. The imaging in the NIR-SWIR range was performed using one Specim FX17 camera (Konica Minolta Company, Oulu, Finland). It captures at a rate of $25 \mathrm{fps}$ in the NIR to SWIR range of the spectrum (1000-1700 nm) with a resolution of 640 pixels per line scan, in 112 wavelength bands across the camera's spectral range. The effective pixel size is $18.7 \times 18.7 \mu \mathrm{m}$, and the spectral resolution is $8 \mathrm{~nm}$.

To simulate an aquatic environment, a black polypropylene container was filled with tap water to a surface level of 50 centimetres below the camera lenses. The black container was used to hold the water and samples since it had negligible reflectance values relative to the reflectance values of the observed items over the entire spectrum. A white sheet of optical grade Spectralon was used as a white reference, having the highest diffuse 130 reflectance of any known material in the range of the spectrum used in this study. The dark reference was captured by completely closing the aperture of the camera, leading to no light striking the sensor resulting in a true dark reference. To ensure consistent illumination of the items, two full-spectrum halogen lamps illuminated the samples at roughly 15 degrees off-axis of the cameras. Both lamp's focuses were aligned at the focus point of the line capture of both cameras. A computer with software was used to capture the data, control the integration time and exposure, and operate the motors for the linear scanner. Both cameras captured at a $0^{\circ}$ - 


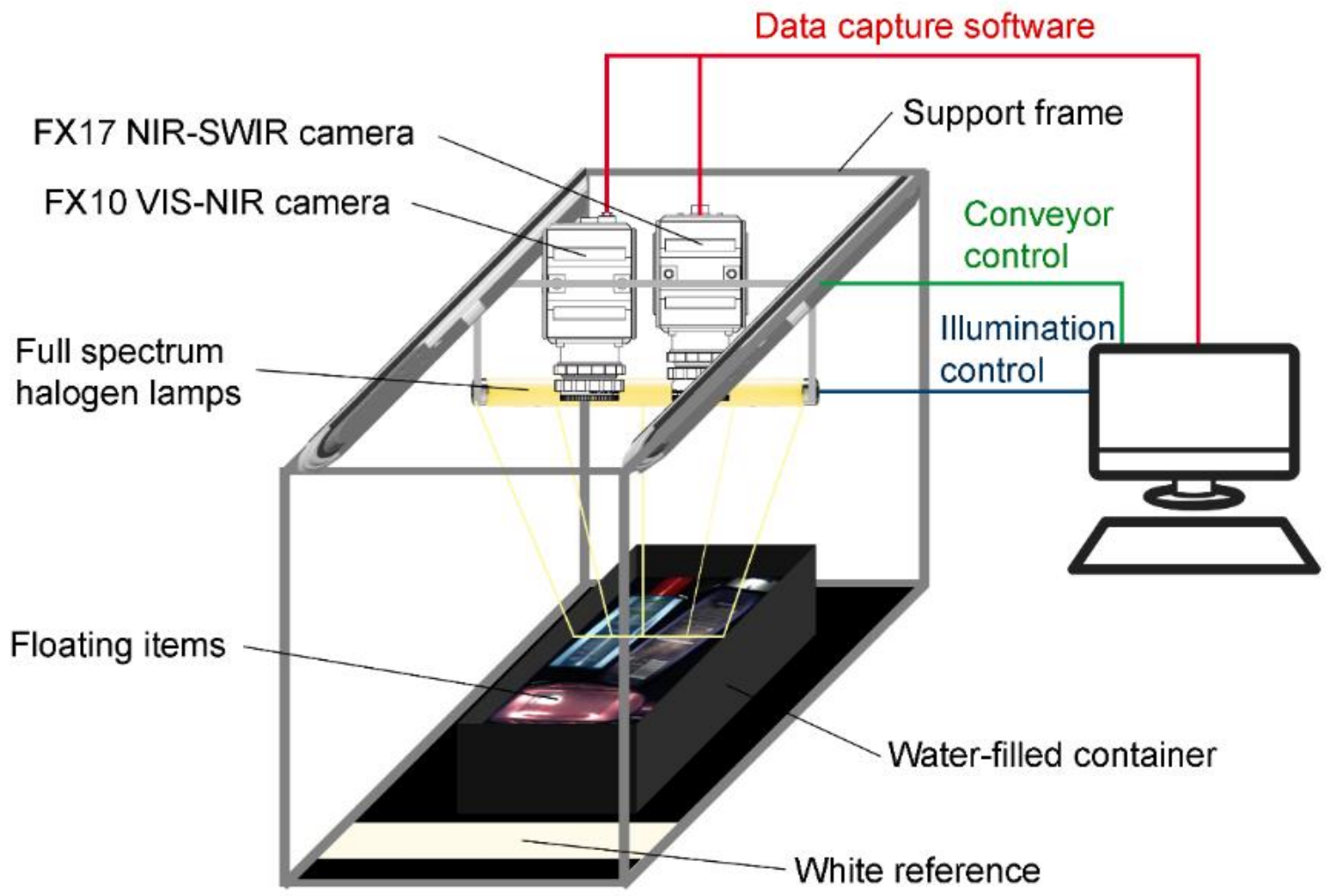

Figure 2. Schematic of the experimental setup

For each image, first, the FX10 camera was centred to scan across the scene. After the final line scan, the white and dark references were captured. Next, the FX17 camera is centred and the procedure for capturing data is repeated, resulting in a 3D spatial-spectral dataset of 1024 (pixels) by 760 (lines) by 224 (bands) and 640 142 (pixels) by 760 (lines) by 112 (bands) for the FX10 and FX17 cameras, respectively. The integration time of the 143 cameras was set to a value between 4 and 8 milliseconds depending on the brightness of the items, minimising 144 overexposure, and maximising the signal-to-noise ratio. Yet, some glare from highly reflective plastic surfaces 145 was inevitable and resulted in small patches of local overexposure. The glare patches were ignored in selecting 146 representative pixels for each item, and the low signal-to-noise ratio did not affect reflections at the lower end 147 of the dataset. Each capture including the white and black reference took approximately 30 seconds per camera, with five seconds in between the captures to return to the starting position.

\subsection{Data preparation}

To further optimise the signal-to-noise ratio, the raw reflectance data were converted into relative reflectance. This was done by using the mean raw reflectance values of the white and dark reference sheets, following the same approach as Zhao et al. [36], referring to the following equation:

$R_{n}=\left(R_{0}-R_{B}\right) /\left(R_{W}-R_{B}\right)$

in which $R_{n}$ is the corrected relative reflectance dataset, $R_{0}$ the raw reflectance dataset, $R_{B}$ the mean dark 
reflectance signatures of the item samples, the lowest and highest bands within the range of both cameras were

omitted.

It is important to note that the cameras have lower spectral response values at both extremes of their range. In

The next step involved normalising the intensity values of all pixels in the dataset, equalising the darker and

in which $R_{n i}$ is the normalised intensity dataset, and $\min \left(R_{n}\right)$ and $\max \left(R_{n}\right)$ the minimum and the maximum item types.

\subsection{Data analysis}

After all virgin plastic items $(n=40)$ and the vegetation were scanned by the hyperspectral cameras, representative pixels for each item were manually selected, using the PerClass machine learning toolbox in MATLAB. Each image was segmented into objects, and each object defined according to an item class, either a type of plastic, vegetation, or water. In total, eight classes were established: (1) water, (2) vegetation, (3) PP, (4) HDPE, (5) LDPE, (6) PET, (7) PS and (8) all plastics combined. For each class, the normalised and averaged spectral signatures of the plastic collection were calculated.

The standard deviation of each averaged spectral signature was calculated to account for the large number of representative pixels (20 - 95 million) per item category. The complete spectral library for the plastic items consists of a different number of pixels counts per item category. Due to the difference in the image dimensions of the two cameras, the representative pixel totals also vary between the VIS-NIR and NIR-SWIR parts of the spectrum. An overview of the representative pixel counts per item category and camera is found in the supplementary material. In total, 1.89 million pixels were sampled per wavelength for all categories in the entire range of the spectrum. This resulted in a database with a total of nearly 6 million pixels, each pixel being a single value in the normalised intensity dataset $R_{n i}$.

The resulting eight classes were used in a data pipeline to (i) extract the spectral signatures of each item class, and (ii) conduct a Fisher linear discriminant analysis (LDA) to find diagnostic features in the spectral signatures. 
variance $\left(\mathrm{S}_{\mathrm{B}}\right)$ is calculated, expressed as the differences in mean values $\left(\mu_{c}-\bar{x}\right)$ of the two classes (Equation

$S_{B}=\sum_{c} \sum_{i \in c}\left(x_{i}-\mu_{c}\right)\left(x_{i}-\mu_{c}\right)^{T}$

$J(v)=\frac{v^{T} S_{B} v}{v^{T} S_{w} v}$

Next, comparisons were made between the weights of the LDA describing the relative contribution of each wavelength to the total variance and the reflectance signatures of those classes. It is important to treat the LDAs from the VIS-NIR and the LDAs from the NIR-SWIR as two separate analyses. Thus, the weights in the VISNIR part are solely based on data acquired by the VIS-NIR camera, and not influenced by measurements in the NIR-SWIR and vice versa. By using all wavelengths of two different classes in the normalised intensity dataset as input for the LDA, a vector with the relative importance of each wavelength expressed as weights is created. Wavelengths with the largest weights resulting from the LDA are considered as diagnostic features, provided the wavelengths align with spectral features characteristic to the item that is analysed. All the materials and MATLAB scripts of the analyses are available in the supplementary material of this paper.

\subsection{Satellite multispectral indices for floating debris}

Using Sentinel-2 multispectral data, the Floating Debris Index (FDI) showed that the difference in spectral signatures of floating materials and water results in the ability to distinguish between the two on sub-pixel scales. detect differences between plastics, vegetation, driftwood and seafoam [17]. To show how these indices work on high-resolution images, they were applied to two hyperspectral images in our lab. The NDVI and FDI were calculated from the normalised intensity dataset. The following equations describe these indices:

in which B4, B6, B8 and B11 are spatial data captured by Sentinel-2's bands at $665 \mathrm{~nm}\left(\lambda_{B 4}\right), 740 \mathrm{~nm}, 842 \mathrm{~nm}$ close to the central bandwidths of the Sentinel-2 bands used in these indices: $666.21 \mathrm{~nm}, 739.64 \mathrm{~nm}, 841.28$ $\mathrm{nm}$, and $1610 \mathrm{~nm}$ for B4, B6, B8, and B11, respectively. An overview of Sentinel-2 Multispectral Instrument 
Table 2: Summary of Sentinel-2 bands and the closest wavelengths in the hyperspectral dataset with their band index number.

\begin{tabular}{|c|c|c|c|c|c|c|c|c|c|c|}
\hline $\begin{array}{l}\text { Sentinel-2 } \\
\text { band name }\end{array}$ & $\begin{array}{l}\text { B2 } \\
\text { blue }\end{array}$ & $\begin{array}{l}\text { B3 } \\
\text { Green }\end{array}$ & B4 Red & $\begin{array}{l}\text { B5 } \\
\text { Veg. } \\
\text { red } \\
\text { edge }\end{array}$ & $\begin{array}{l}\text { B6 } \\
\text { Veg. } \\
\text { red } \\
\text { edge }\end{array}$ & $\begin{array}{l}\text { B7 } \\
\text { Veg. } \\
\text { red } \\
\text { edge }\end{array}$ & B8 NIR & $\begin{array}{l}\text { B9 } \\
\text { Water } \\
\text { vapour }\end{array}$ & $\begin{array}{l}\text { B10 } \\
\text { SWIR- } \\
\text { Cirrus }\end{array}$ & $\begin{array}{l}\text { B11 } \\
\text { SWIR }\end{array}$ \\
\hline $\begin{array}{l}\text { Central } \\
\text { wavelength } \\
(\mathrm{nm})\end{array}$ & 490 & 560 & 665 & 705 & 740 & 783 & 842 & 945 & 1375 & 1610 \\
\hline $\begin{array}{l}\text { Hyperspectral } \\
\text { data } \\
\text { wavelength }\end{array}$ & 489.3 & 561.2 & 666.2 & 704.2 & 739.6 & 783.5 & 841.28 & 941.1 & 1377.4 & 1610.6 \\
\hline Band number & vis-36 & vis-63 & vis-102 & vis-116 & vis-129 & vis-145 & vis-166 & ir-1 & ir-63 & ir-96 \\
\hline
\end{tabular}

Table 3: Summary of Worldview-3 bands and the closest wavelengths in the hyperspectral dataset with their band index number.

\begin{tabular}{lllllllllll}
\hline $\begin{array}{l}\text { Worldview-3 } \\
\text { band name }\end{array}$ & Blue & Green & Yellow & Red & $\begin{array}{l}\text { Red } \\
\text { edge }\end{array}$ & $\begin{array}{l}\text { Near- } \\
\text { IR1 }\end{array}$ & $\begin{array}{l}\text { Near- } \\
\text { IR2 }\end{array}$ & $\begin{array}{l}\text { SWIR- } \\
\mathbf{1}\end{array}$ & $\begin{array}{l}\text { SWIR- } \\
\mathbf{2}\end{array}$ & $\begin{array}{l}\text { SWIR- } \\
\mathbf{3}\end{array}$ \\
\hline $\begin{array}{l}\text { Central } \\
\text { wavelength } \\
(\mathrm{nm})\end{array}$ & 480 & 545 & 605 & 660 & 725 & 833 & 950 & 1219 & 1570 & 1660 \\
$\begin{array}{l}\text { Hyperspectral } \\
\text { data } \\
\text { wavelength }\end{array}$ & 481.4 & 545.2 & 604.1 & 660.8 & 726.0 & 833.0 & 948.1 & 1222.3 & 1568.1 & 1660.2 \\
$\begin{array}{l}\text { Band number } \\
\text { vis-33 }\end{array}$ & vis-57 & vis-79 & vis-100 & vis-124 & vis-163 & ir-2 & ir-41 & ir-90 & ir-103 \\
\hline
\end{tabular}

In this section, three main findings are presented and discussed. First, we present an in-depth analysis of the 229 spectral signatures to build an understanding for reflectance-based item identification. Second, the results of 230 the linear discriminant analysis are shown to demonstrate how certain wavelengths are more useful for 231 discriminating between water, vegetation, and plastics. Third, we compare our results with current satellite232 based indices used for plastic detection.

\subsection{Spectral analyses}

Water, vegetation and the five types of plastic each show unique spectral signatures. Unsurprisingly, the reflectance of water in the NIR-SWIR range $(950-1700 \mathrm{~nm})$ is consistently close to zero, whereas plastics and vegetation are characterised by higher reflectance values and unique absorption peaks in this range (Figure 3a-h). For instance, the average spectral signature for all plastics combined shows two distinct absorption peaks at $1215 \mathrm{~nm}$ and $1410 \mathrm{~nm}$ (Figure 3c). As opposed to plastics, vegetation is characterised by a stronger 239 absorption peak at $1450 \mathrm{~nm}$. 
(a) WATER ( $n=408,879)$

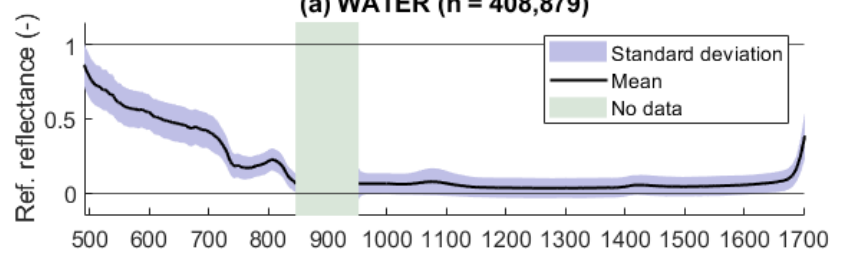

(c) PLASTIC (combined) $(n=189,126)$

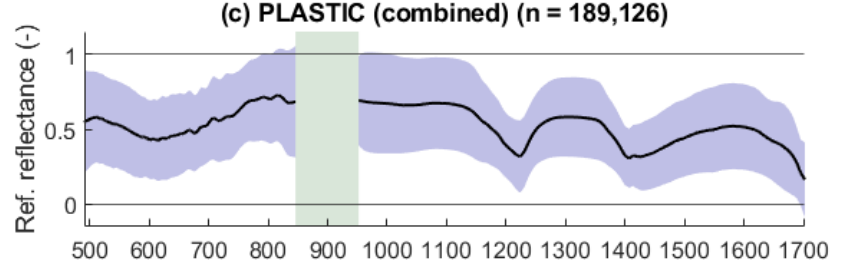

(e) HDPE ( $n=351,985)$

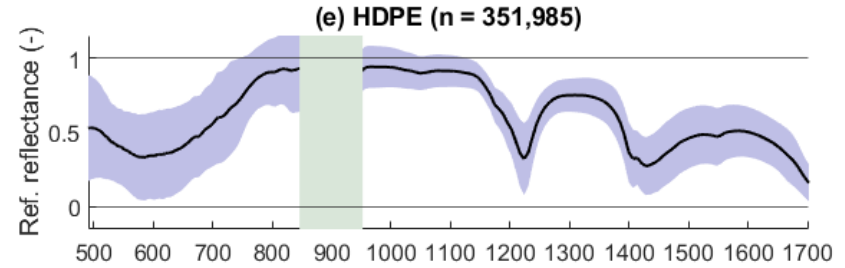

(g) PET ( $n=225,842$ )

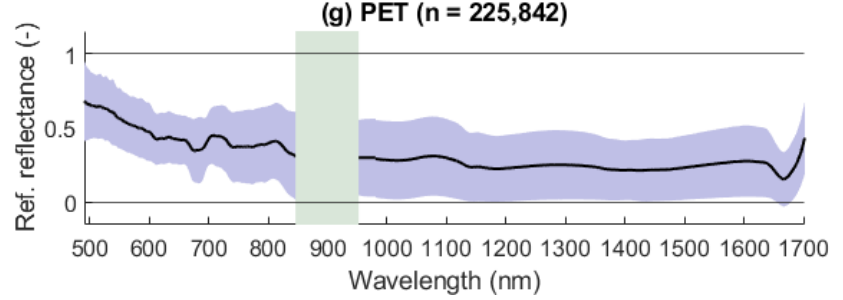

(b) VEGETATION ( $\mathrm{n}=\mathbf{2 0 6 , 0 8 3}$ )

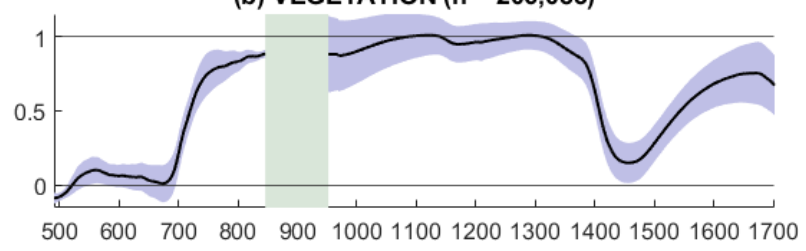

(d) PP ( $n=189,126)$

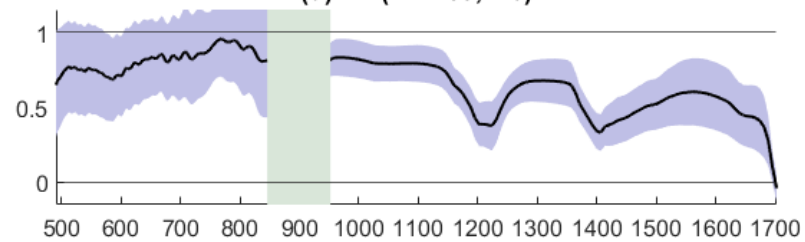

(f) LDPE ( $n=190,664)$

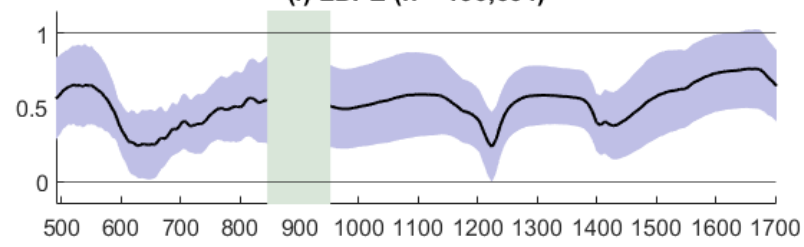

(h) PS ( $n=85,726)$

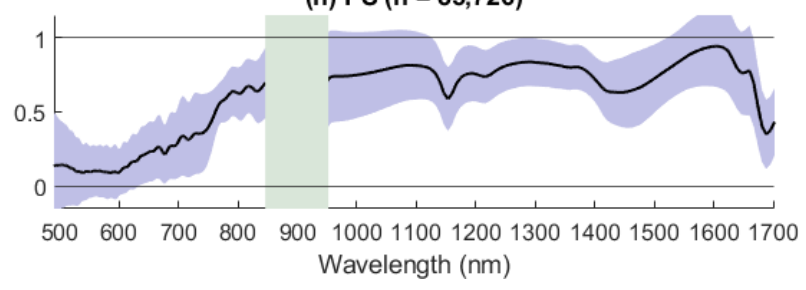

Figure 3: Normalised spectral signatures of the virgin plastic collection, water, and vegetation. The spectral signatures were calculated

The standard deviation of all plastics combined is relatively high in comparison to the standard deviation of vegetation and water, which is likely caused by the large number of pixels ( $n=1.27$ million) and the differences in the reflection spectra of the individual polymer types (Figure 3). In contrast, water and vegetation have smaller standard deviations across the full spectral range. PP and HDPE show smaller variability in the NIR-SWIR range than they do in the VIS-NIR range. The other classes of LDPE, PET, PS, and combined plastics (Figure 3c, $f$ h) show high variability across the full spectral range. From Figure $3 g$ and $3 f$, it is clear that PET and PS do not have strong absorption features at the typical plastic absorption peak around $1215 \mathrm{~nm}$.

High density and low-density polyethylene (HDPE and LDPE) polymers have similar absorption features in the

950-1700 nm range of the spectrum, where reflectance in this range is driven by material properties, rather than the apparent colour of objects [40]. The two absorption features of polyethylene (PE) plastics are centered around 1210 and $1430 \mathrm{~nm}$. Polypropylene (PP) plastics show clear absorption features at 1205 and $1400 \mathrm{~nm}$, which are in close correspondence with Moshtaghi et al. [24]. Polystyrene (PS) is characterised by two distinct absorption features at 1150 and $1450 \mathrm{~nm}$. Moroni et al. [41] found strong absorption features at $1130 \mathrm{~nm}, 1170$ $\mathrm{nm}, 1420 \mathrm{~nm}$, and $1660 \mathrm{~nm}$ for aggregated opaque PET granules, whereas a nearly flat spectral signature is found for transparent samples. In fact, the spectral shape of the transparent PET samples found by Moroni et al. [41] is similar to the spectral shape found in this study (Figure 3g). From this, we hypothesise the lack of characteristic PET absorption features in Figure $3 g$ is due to the transparency of the PET samples and that spectral signatures of non-transparent PET items might yield different results. 
Overall, the absorption features of the polymer types shown in Figure 3 show good agreement with absorption features of the same reflection signatures documented in other studies [25, 27, 40]. For plastics, a typical absorption peak is centered around $1215 \mathrm{~nm}$, which is commonly referred to in other studies [13, 40]. On a molecular level, this typical absorption characteristic of HDPE, LDPE and PP peak is due to the second overtone of Carbon-Hydrogen (C-H) stretching [42]. When a plastic sample is illuminated by light, specific atomic groups, such as $\mathrm{C}-\mathrm{H}$ and Oxygen-Hydrogen $(\mathrm{O}-\mathrm{H})$ can absorb energy, resulting in a change of vibrational energy state. Hensold et al. [43]. The characteristic rise in spectral reflectance in the 700-720 nm range is caused by the internal cell structure of leaves, while the absorption feature around $1450 \mathrm{~nm}$ is caused by leaf water content [44].

At specific wavelengths in the spectrum, overtone bands are present, indicating when a molecule switches from its lowest energy state (ground state) to an excited state with a greater energy level. The first and second overtone occur in the NIR-SWIR range of the spectrum and are characterised by the largest jumps in the energy level. This implies these overtones are characterised with stronger absorption features than the third, fourth and fifth overtones, which are positioned in the VIS-NIR range of the spectrum. The second absorption peak at 1410 $\mathrm{nm}$ - noticeable for all plastic items except PET - at $1410 \mathrm{~nm}$ is in also in line with results of other studies and appears to be caused by the first overtone of $\mathrm{O}-\mathrm{H}$ stretching vibration, characteristic for multiple plastic polymer configurations $[13,25,45]$.

Just beyond the spectral range used in this study, an absorption peak at $1747 \mathrm{~nm}$ was previously found for multiple plastic types, which can be attributed to the first overtone of $\mathrm{C}-\mathrm{H}$ stretching [45]. Additionally, multiple studies indicated small absorption features for plastics to be present in the missing range used in this study (850-950 nm), which are primarily attributable to the third overtone of $\mathrm{C}-\mathrm{H}$ stretching $[13,23,46]$. Further research on the spectral ranges beyond $1700 \mathrm{~nm}$, and between $850-950 \mathrm{~nm}$ are needed to provide a complete overview of the distinct spectral signatures of various plastic items.

\subsection{Linear discriminant analyses and comparison with satellite remote sensing approaches}

\subsubsection{Separating floating debris from water}

The distinctly different spectral signatures of water and floating items resulted in a range of low and high LDA weights overlapping with multiple satellite bands. Figure $4 a$ shows the spectral signature of all floating items combined compared with the spectral signature of water. The absolute difference between floating item reflectance values and water reflectance values in the SWIR range is notable. Moreover, the reflection signature of water is close to zero and nearly flat over the entire range in the NIR-SWIR. Figure $4 \mathrm{~b}$ and $4 \mathrm{c}$ show the weights of the LDA describing the power of each wavelength in discriminating between water and floating items. Each wavelength in the spectrum has a different importance for distinguishing between floating items and water (Figure 4). Additionally, the central wavelengths and their bandwidths of the Sentinel-2 satellite (Figure 4b) and the Worldview-3 satellite (Figure 4c) are shown. It is apparent that some of the central wavelengths are overlapping with relatively high LDA weights, whereas other bands seem to be less important for separating classes. 
(a) Spectral reflectance - Water \& Floating items

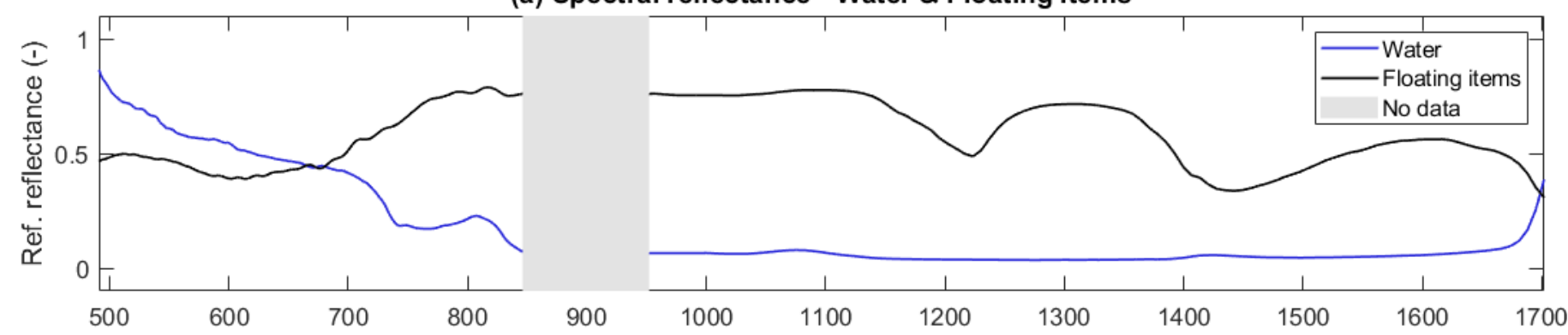

(b) Sentinel-2 bands \& LDA weights

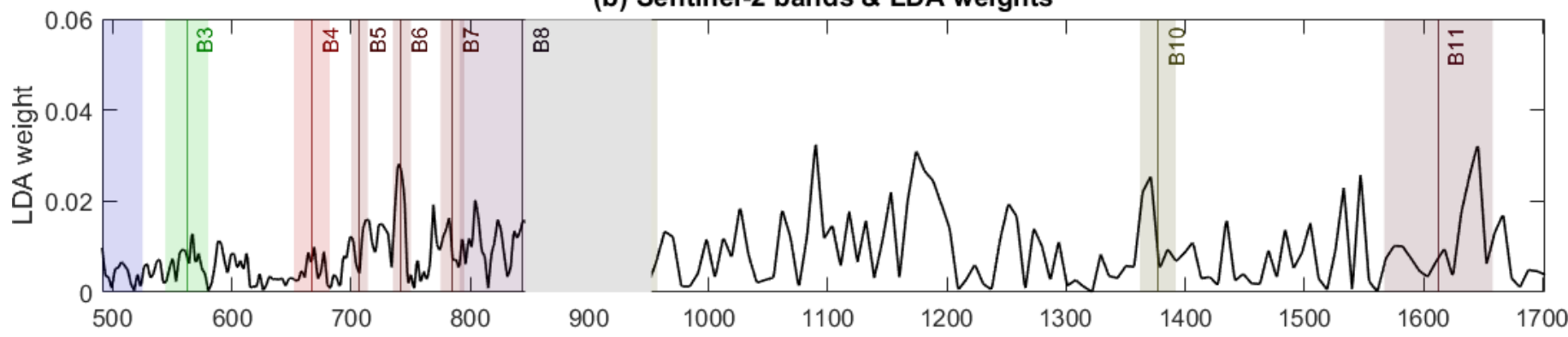

(c) Worldview-3 bands \& LDA weights

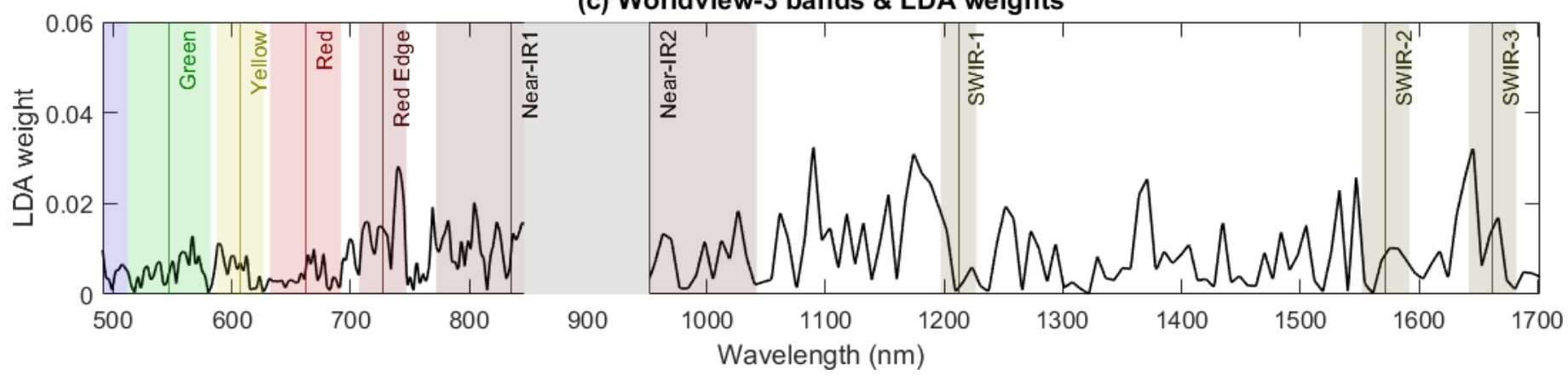

Figure 4: Reflection spectra of water and floating items (a); weights of the LDA separating water and floating items overlaid with central

wavelengths and bandwidths of the Sentinel-2 MSI (b); and overlaid with central wavelengths and bandwidths of Worldview-3 MS-VNIR \&

MS-SWIR (c).

As is shown Figure 4b, Sentinel-2 bands B6 (red edge), B10 and B11 (SWIR) are most powerful for discriminating between water and floating debris, though B10 is a lower spatial resolution band predominantly used for cirrus detection [47]. The data gap in the NIR prevents us from fully observing to what extent Sentinel303 2 B8 and B8A are important, however Biermann et al. [17] suggest B8 is key for detection of floating debris. For 304 Worldview-3, the Red Edge and SWIR-3 overlap with highest weights (Figure 4C). There are no clear features in the spectra explaining why these bands have high LDA weights. In their study, Guo and Li [48] suggest SWIR306 2, SWIR-3 and SWIR-4 are important for detecting plastic surfaces in urban areas. Since water is much more 307 uniform than diverse urban areas, it is feasible these bands will also work for detection of floating plastics. 
The unique spectral signatures of plastic and vegetation allowed an identification of several important wavelengths, overlapping with multiple Sentinel-2 and Worldview-3 bands. In Figure 5a, the reflectance spectra and Worldview-3 bands, respectively.

(a) Spectral reflectance - Vegetation \& Plastics

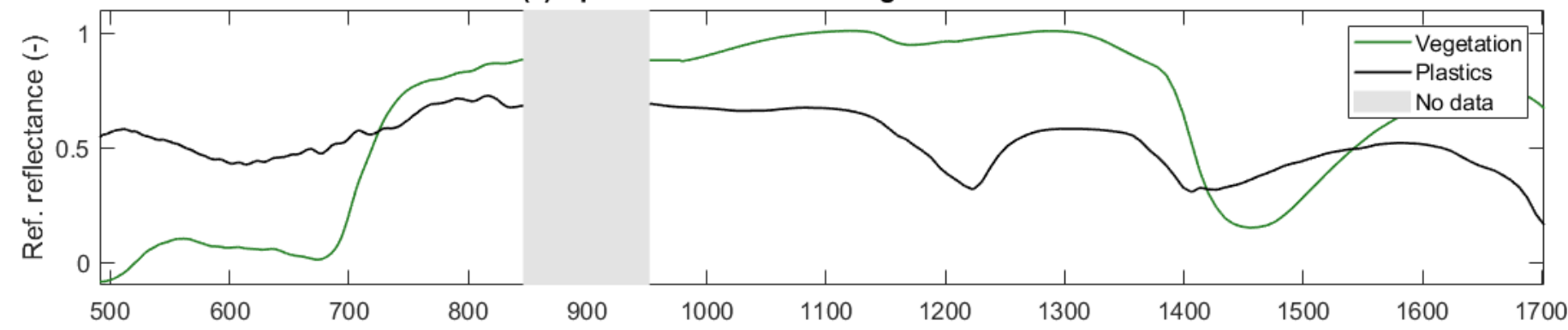

(b) Sentinel-2 bands \& LDA weights

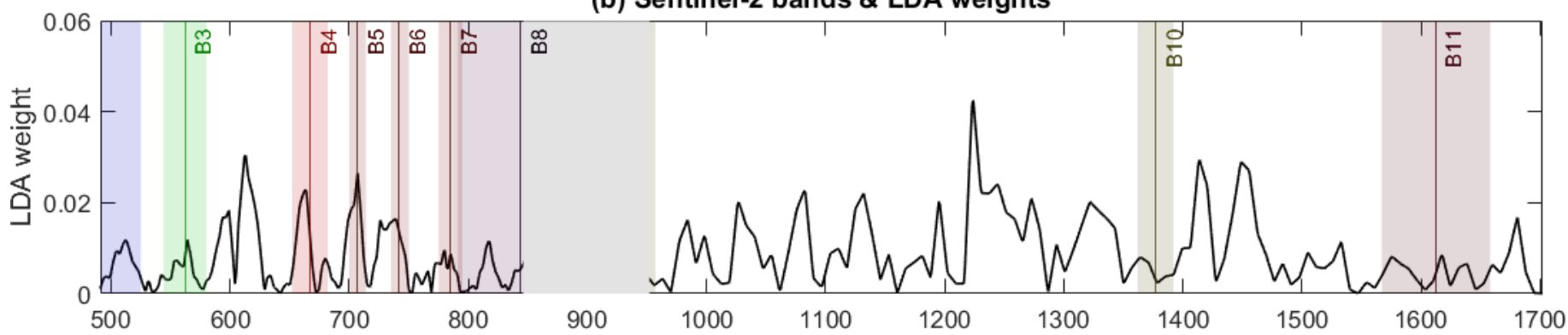

(c) Worldview-3 bands \& LDA weights

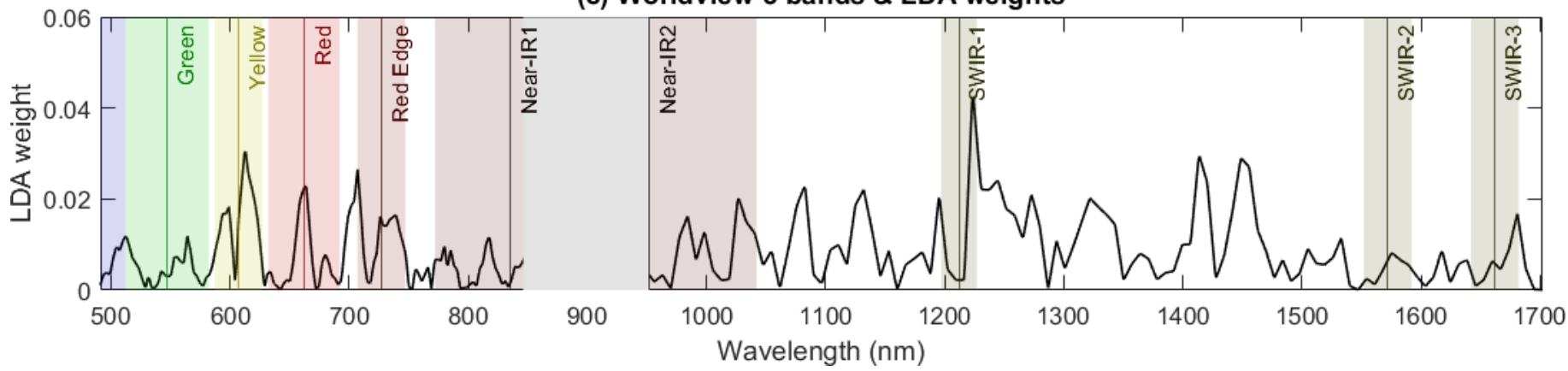

Figure 5: Reflection spectra of vegetation and plastics (a); weights of the LDA separating vegetation and plastics items overlaid with central 314 wavelengths and bandwidths of the Sentinel-2 MSI (b); and overlaid with central wavelengths and bandwidths of Worldview-3 MS-VNIR \& MS-SWIR (c).

In the VIS-NIR part of the spectrum, two wavelengths centred around $615 \mathrm{~nm}$ and $710 \mathrm{~nm}$ have the highest weights. The high LDA weight at $615 \mathrm{~nm}$ lines up with the lowest reflection value of plastic items in the VIS-

SWIR range. The importance of the reflection at $615 \mathrm{~nm}$ is strongly influenced by the apparent colour of floating objects [40], which makes the importance of this wavelength debatable. A different collection of sample items, in combination with a relatively large standard deviation, could easily result in a different set of wavelengths having the high LDA weights. The high weight at $710 \mathrm{~nm}$ occurs in the red edge (red-NIR transition of vegetation reflectance). The red edge bandwidth delineates between chlorophyll absorption in the red wavelengths of light, 
and reflection in the NIR due to the cellular structure of vegetation. Thus, the high weight at this wavelength appears to be driven by vegetation-specific reflectance.

In the NIR-SWIR range of the spectrum, important wavelengths are centred around $1215 \mathrm{~nm}, 1410 \mathrm{~nm}$, and 326 $1450 \mathrm{~nm}$, which can be attributed to the absorption peaks of both plastic and vegetation. The absorption peak of PS around $1140 \mathrm{~nm}$ is barely reflected at all in the relative importance of this wavelength according to the LDA weights. Whilst the reflectance of other plastics is lowest at $1215 \mathrm{~nm}$, the reflectance of PS is already past 329 its absorption peak. This makes it so the importance of the rise in PS reflectance between 1140-1200 nm is 330 cancelled out by the steadily declining reflectance of the other plastics in this range. The other important wavelengths in the NIR-SWIR range are centred around $1410 \mathrm{~nm}$ and $1450 \mathrm{~nm}$, which can be attributed to the 332 absorption peaks of plastic and vegetation, respectively.

For the Sentinel-2 MSI, bands B4 $(665 \mathrm{~nm})$, B5 $(705 \mathrm{~nm})$, and B6 $(740 \mathrm{~nm})$ have high LDA weights, implying 334 these bands are promising for separating vegetation and plastics. The reflection spectrum of plastics in the VIS$\mathrm{NIR}$ (Figure 3c) does not show unique features, but the vegetation spectrum is characterised by the formerly explained chlorophyll absorption and cellular structure in this range. The high LDA weight at $1215 \mathrm{~nm}$ caused by the absorption peak of plastics is not captured by any existing Sentinel-2 band. Therefore, new sensors with a band at a central wavelength of $1215 \mathrm{~nm}$ would be key for future ESA missions focussed on plastic-specific detection.

For Worldview-3 however, the high LDA weight of the absorption feature at $1215 \mathrm{~nm}$ lines up with the bandwidth of SWIR-1. Additionally, the 'Yellow', 'Red' and 'Red Edge' bands of Worldview-3 are also characterised by high weights when distinguishing between plastics and vegetation. Combined with the higher spectral resolution offered by the worldview satellites (1.24 $\mathrm{m}$ for MS-NIR and $3.7 \mathrm{~m}$ for MS-SWIR), these low-orbit satellite sensors may prove well suited for detecting plastic, specifically.

\subsubsection{Comparison of satellite-derived products with LDA results}

As illustrated in Figure 4 and 5, all the bands used in the NDVI and FDI, referring to equations 3 and 4, are associated with a range of different LDA weights. It is apparent that Sentinel-2's B4, B6, B8 and B11 have different weights when separating water and floating items, compared to the weights when separating vegetation and plastics. For example, B4 stands out for vegetation and plastics separation, whereas this band has a much lower weight when separating water and floating debris. This is one of the reasons why the NDVI is suitable for 350 highlighting vegetation. B5 $(705 \mathrm{~nm})$ and B6 $(740 \mathrm{~nm})$ have high LDA weights when separating plastics and 351 vegetation (Figure $5 b)$. Since these bands are not used in the NDVI, and B8 $(842 \mathrm{~nm})$ is important for highlighting all floating items, the NDVI is to some extent capable of highlighting certain types of plastic in addition to vegetation, as is seen in Figure $6 \mathrm{e}$.

For the FDI, designed for detecting floating debris, B6 $(740 \mathrm{~nm})$ and B8 $(842 \mathrm{~nm})$ bands are associated with high LDA weights of 0.28 and 0.16 , respectively. These bands appear to be the main reason why the FDI wavelengths. Even though the central wavelength and LDA weight of B8 falls within the spectral range of this study, the full bandwidth of B8 is not captured because of the lack of data between 850 and $950 \mathrm{~nm}$. Therefore, 
the LDA result of $B 8$ could be incomplete when considering its full bandwidth range. The $B 4(665 \mathrm{~nm})$ and $B 11$

$(1610 \mathrm{~nm})$ bands have significantly lower LDA weights than the other bands. However, it is likely B11 contributes

From Figure $6 \mathrm{~b}$ and $6 \mathrm{e}$, it is clear that healthy vegetation results in high NDVI values. However, the intensity values are not always high for plastics. Parts of the LDPE bag in Figure $6 e$ are characterised by high intensity values, but the pixels in the lower right part of the image have much lower values. Moreover, the PP spoons and PS coffee stirrers in Figure $6 \mathrm{~b}$ are not highlighted at all by the NDVI. The FDI on the other hand can highlight all three plastic types and vegetation, as seen in Figure $6 \mathrm{c}$ and $6 \mathrm{f}$. A drawback of this index is its response to a thin layer of water covering the floating item. In fact, when a sample is only slightly submerged by water, the FDI approaches zero, as is evident in Figure 6c. This is likely caused by enhanced water absorption in the NIR366 SWIR, which results in the apparent disappearance of spectral features when items are submerged [13]. Figure 6 provides just two examples, and we highly encourage future studies to explore all hyperspectral data provided in the supplementary material, using either existing or new indices.
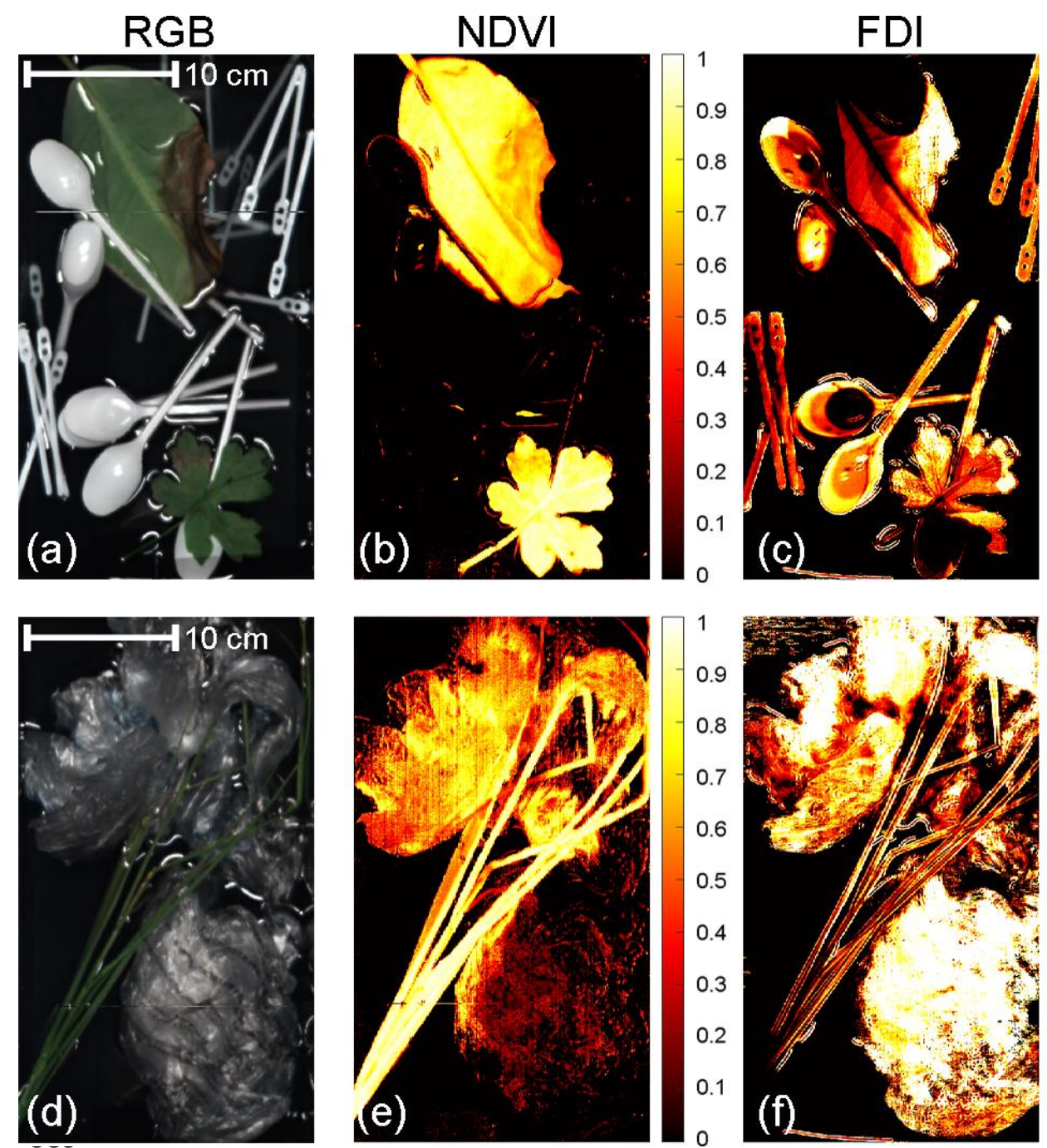

Figure 6: Two examples of the Normalised Difference Vegetation Index (NDVI) (b, e), and Floating Debris Index (FDI) (c, f) with their 
A combination of the NDVI and FDI is perhaps the most robust approach for separating plastics and vegetation.

Figure 7 shows a scatterplot of the NDVI versus the FDI for water, vegetation, and the five polymer categories.

It also shows the range of water, plastic, and vegetation values as found by Biermann et al. [17]. Water has a low mean value for both indices, whereas healthy vegetation has the highest mean value. The vegetation has a significantly higher mean value in the NDVI compared to Biermann et al. [17], which is likely caused by the selection of the most green (healthy) pixels, as opposed to floating seaweed. The different plastic types have overlapping standard deviations but are relatively different from water and vegetation. Floating aquatic vegetation and riverine vegetation typically have lower NDVI values, ranging from 0.4 to 0.7 , which results in an overlap with NDVI values of several polymer types [49]. This in turn hampers discrimination between the specific 400 polymers and vegetation based on NDVI alone. Using a combination of the NDVI and FDI for Sentinel-2 based detection of debris is a powerful approach for detecting patches of mixed debris with plastics, floating on and 402 just below the surface. The FDI cannot be exactly replicated with the bands offered by the Worldview satellites, 403 but indices that leveraged the red edge and SWIR-3 bands for debris detection, and the yellow and SWIR-1 bands for plastic discrimination would likely produce promising results.

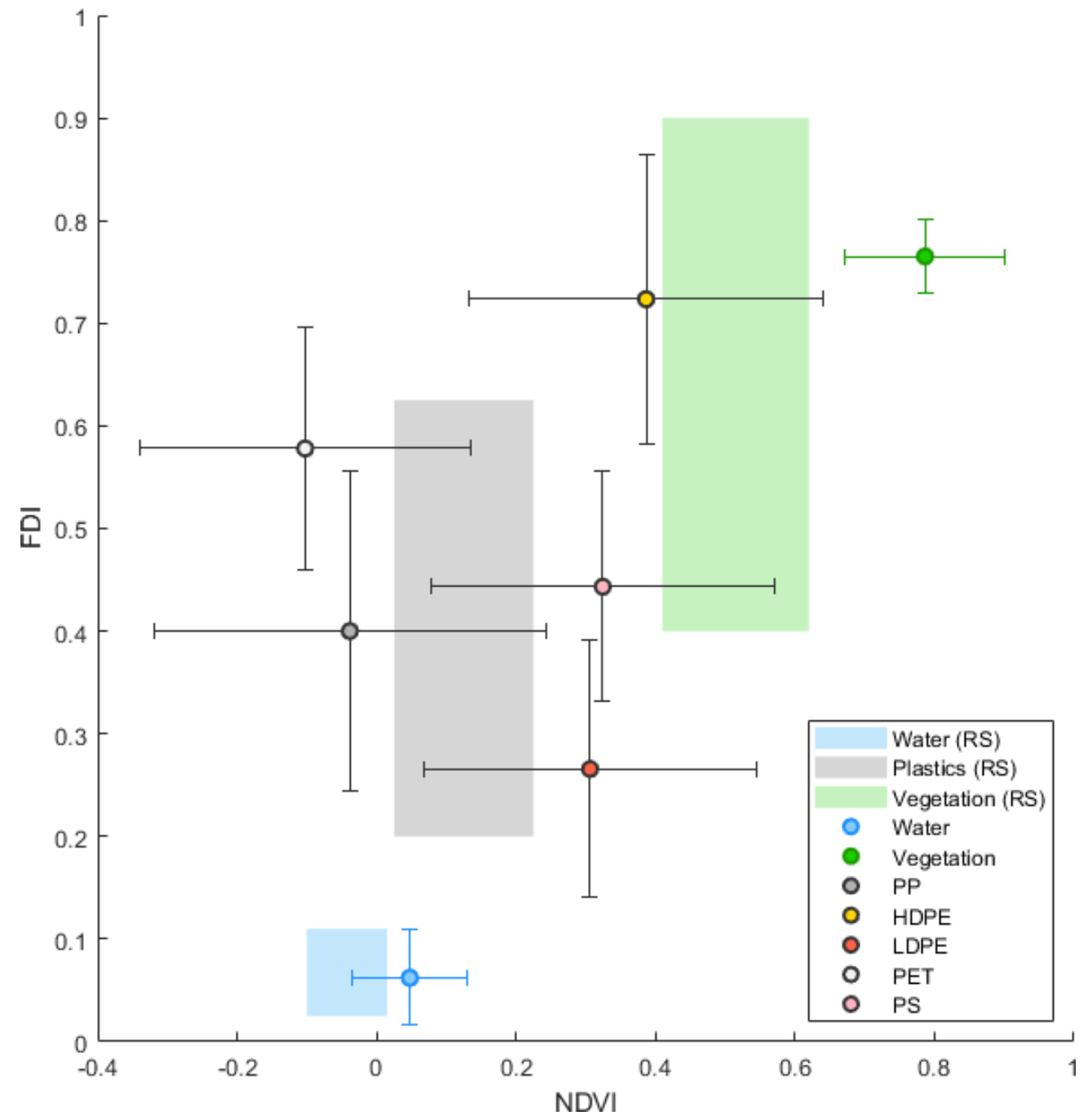

Figure 7: FDI and NDVI scatter for all seven classes with their mean value (coloured dots) and standard deviation as error bars. The 
Hyperspectral imaging systems have a great potential for reflection-based macroplastic detection and identification. Using these systems in airborne or spaceborne remote sensing tools can help identifying plastic waste in heavily polluted rivers, on beaches, and in remote marine environments. So far, several studies have explored spectral signatures of floating macroplastics, either with spectroradiometers or in combination with high resolution imagery. Recent efforts mainly include measurements in controlled environments [23-25, 27], focussing on full-spectrum characterisation of reflectance signatures. Garaba and Dierssen [13] established an open-access database of spectral signatures of marine-harvested and washed-ashore plastics, identifying absorption features of various plastic samples. Goddijn-Murphy and Dufaur [20] used hyperspectral data in an optical reflectance model as a step towards automatic detection of floating marine plastic litter. Corbari et al. [25] spectrally characterised various polymer types and attempted to quantify optimal band combinations for multispectral monitoring of plastics. Next to these studies, the dataset presented in our study provides some of the first fundamental baselines in the rapidly developing field of hyperspectral imaging.

Similar to other studies, we spectrally characterised various floating macroplastic samples in a controlled environment. A unique addition of our study are in-depth linear discriminant analyses, to demonstrate which wavelengths are optimal for separating water and floating objects, and vegetation and plastics. In addition to Corbari et al. [25], we used high-resolution imagery to validate current multispectral remote sensing techniques, next to identifying which bands are optimal for multispectral plastic monitoring. In doing so, we have supported the importance of combining the NDVI and FDI for plastic detection using Sentinel-2 data. We have also identified absorption peaks for plastics at $1215 \mathrm{~nm}$ and $1410 \mathrm{~nm}$, which should be considered in the development of future multispectral sensors for plastic detection.

Only few studies have conducted hyperspectral measurements of plastics on natural surfaces, either UAVbased in coastal and marine environments [50] or in a controlled environment with natural sunlight [13]. However, most studies performed outside are limited to multispectral imaging systems, having between six and twelve bands, as opposed to over a hundred in hyperspectral studies. Most of these studies use airborne or spaceborne multispectral data [15-17, 21], often successfully detecting and identifying plastic targets with satellite-derived feature extraction indices. In our work, we highlighted which bands should be included in the limited six to twelve bands of multispectral sensors for better detection and identification of floating plastics.

We present a novel hyperspectral reflectance database of common plastic litter items used to validate current satellite techniques for plastic detection and identification. This open-access database is unique compared to 438 other reference libraries, as it provides high-resolution imagery in over 300 spectral bands. The database can be explored to get a detailed understanding of satellite remote sensing and their derived products, but also where future improvements could be made. Due to the missing data between 850-950 nm in this study, we were not able to fully explore the role of several Sentinel-2 bands (B8, B8A) and Worldview-3 bands (NIR 1, NIR 2) in detection of floating debris. We highly encourage future studies using hyperspectral imagery to work towards characterising absorption features beyond the spectral range and limitations of this study, to establish their importance for new multispectral sensor setups. As a baseline for future studies, all data and code used for our 
Our hyperspectral reference dataset enables multiple future research directions. First, pixels from the dataset could be aggregated to lower resolutions, representing the mixed nature of sampled pixels by satellite sensors. Floating plastic patches smaller than the pixel size of current satellite-acquired imagery can indeed be detected [16]. A hyperspectral understanding of these mixed pixels could lead to new insights into highly sensitive wavelengths for detection of floating plastic debris. The latter may include the development of new airborne or space-borne multispectral sensors based on the most discriminating bands separating water and floating items, and plastics and vegetation (Figure 4 \& Figure 5).

Second, hyperspectral classifiers could be built, enabling accurate detection of floating plastics and subsequent polymer-specific classification. For example, Balsi et al. [50] built a classifier algorithm and successfully identified and separated PE and PET plastics. Another application of hyperspectral classifiers is in plastic recycling plants, where mixed plastic waste is sorted using classifier algorithms [51, 52]. Since the five plastic polymer types in our study are characterised by slightly different spectral signatures, a large patch of one specific type of plastic could require a different combination of wavelengths for accurate detection than others. By leveraging the current understanding of hyperspectral-based classifiers, existing remote sensing techniques using multispectral-based extraction indices can be optimised. This enables further contribution to the development of advanced floating debris monitoring and classification missions.

\section{Conclusion}

We present a new hyperspectral database for virgin plastics and vegetation that can be further explored to improve the fundamental understanding of reflectance-based plastic identification. Hyperspectral imaging was done by using a double-camera setup spanning from the visual to shortwave infrared range of the spectrum (400-1700 nm). Future work exploring applications of reflectance-based plastic identification can use this the values in dataset as a baseline.

Second, we identified absorption peaks in the reflectance spectrum of plastics at unique positions in the electromagnetic spectrum (1215 nm and $1410 \mathrm{~nm}$ ). The hyperspectral image database allowed an in-depth

Third, we disclosed the importance of each wavelength in the 400-1700 nm range in separating water from

mixed floating debris, and vegetation from plastic. We conducted linear discriminant analyses of spectral signatures of plastic, water and vegetation and compared the weights of each wavelength with existing bands covered by the Sentinel-2 and Worldview-3 satellites. In doing so, a wider understanding of satellite-derived products such as the NDVI and FDI is created. We found high LDA weights to correspond with the wavelengths of the Sentinel-2 B4, B6 and B8 bands, indicating which components of the NDVI and FDI work best. Using a combination of the NDVI and FDI for Sentinel-2 data or possible indices based on Worldview-3 data is perhaps the most robust approach for detecting patches of mixed floating debris with plastics.

We leveraged the unique properties of reflectance signatures of plastics, vegetation, and water in linear 
improvements of indices based on satellite imagery, a foundation for the development of future multispectral or hyperspectral plastic monitoring missions is built.

Funding: The work of T.v.E is supported by the 4TU.Federation Plantenna project, and the Veni research

Data Availability: All data used for this work are uploaded to the 4TU data repository. A DOI will be provided 489 upon publication of the final manuscript.

Conflicts of Interest: The authors declare no conflict of interest.

\section{References}

1. Gallo, F., et al., Marine litter plastics and microplastics and their toxic chemicals components: the need

2. Borrelle, S.B., et al., Predicted growth in plastic waste exceeds efforts to mitigate plastic pollution.

3. Meijer, L.J., et al., More than 1000 rivers account for $80 \%$ of global riverine plastic emissions into the ocean. Science Advances, 2021. 7(18): p. eaaz5803.

4. Kiessling, T., et al., Plastic Pirates sample litter at rivers in Germany - Riverside litter and litter sources 500 estimated by schoolchildren. Environmental Pollution, 2019. 245: p. 545-557.

5. van Emmerik, T., et al., Crowd-based observations of riverine macroplastic pollution. Frontiers in Earth

6. Gasperi, J., et al., Assessment of floating plastic debris in surface water along the Seine River. 504 Environmental Pollution, 2014. 195: p. 163-166.

7. Lahens, L., et al., Macroplastic and microplastic contamination assessment of a tropical river (Saigon

10. Vriend, P., T. van Emmerik, and C.T. Roebroek, Same but different: $A$ framework to design and compare riverbank plastic monitoring strategies. 2020.

11. Roebroek, C.T.J., et al., Disentangling Variability in Riverbank Macrolitter Observations. Environmental 
12. van Lieshout, C., et al., Automated River Plastic Monitoring Using Deep Learning and Cameras. Earth and Space Science, 2020. 7(8): p. e2019EA000960.

13. Garaba, S.P. and H.M. Dierssen, Hyperspectral ultraviolet to shortwave infrared characteristics of 517 marine-harvested, washed-ashore and virgin plastics. Earth System Science Data, 2020. 12(1): p. 77-86.

14. De Giglio, M., et al., Plastics waste identification in river ecosystems by multispectral proximal sensing:

15. Topouzelis, K., A. Papakonstantinou, and S.P. Garaba, Detection of floating plastics from satellite and unmanned aerial systems (Plastic Litter Project 2018). International Journal of Applied Earth Observation and

17. Biermann, L., et al., Finding Plastic Patches in Coastal Waters using Optical Satellite Data. Scientific 526 Reports, 2020. 10(1): p. 5364.

18. Kikaki, A., et al., Remotely Sensing the Source and Transport of Marine Plastic Debris in Bay Islands of Honduras (Caribbean Sea). Remote Sensing, 2020. 12(11): p. 1727.

19. Garaba, S.P., et al., Sensing Ocean Plastics with an Airborne Hyperspectral Shortwave Infrared Imager.

20. Goddijn-Murphy, L. and J. Dufaur, Proof of concept for a model of light reflectance of plastics floating on natural waters. Marine Pollution Bulletin, 2018. 135: p. 1145-1157.

21. Martínez-Vicente, V., et al., Measuring Marine Plastic Debris from Space: Initial Assessment of 534 Observation Requirements. Remote Sensing, 2019. 11(20).

22. Garaba, S.P. and H.M. Dierssen, An airborne remote sensing case study of synthetic hydrocarbon detection using short wave infrared absorption features identified from marine-harvested macro- and 537 microplastics. Remote Sensing of Environment, 2018. 205: p. 224-235.

23. Knaeps, E., et al., Hyperspectral-reflectance dataset of dry, wet and submerged marine litter. Earth 539 System Science Data, 2021. 13(2): p. 713-730.

24. Moshtaghi, M., et al., Spectral reflectance of marine macroplastics in the VNIR and SWIR measured in a controlled environment. Scientific Reports, 2021. 11(1): p. 1-12.

25. Corbari, L., et al., Indoor spectroradiometric characterization of plastic litters commonly polluting the 543 Mediterranean Sea: toward the application of multispectral imagery. Scientific Reports, 2020. 10(1): p. 1-12.

26. Corbari, L., et al., Indoor spectroradiometric characterization of plastic litters commonly polluting the 
27. Serranti, S., et al., Microplastics characterization by hyperspectral imaging in the SWIR range. SPIE 547

Future Sensing Technologies. Vol. 11197. 2019: SPIE.

28. Topouzelis, K., et al., Remote Sensing of Sea Surface Artificial Floating Plastic Targets with Sentinel-2 549 and Unmanned Aerial Systems (Plastic Litter Project 2019). Remote Sensing, 2020. 12(12). 550

29. Rokni, K., et al., Water Feature Extraction and Change Detection Using Multitemporal Landsat Imagery. 551

Remote Sensing, 2014. 6(5).

30. Lechthaler, S., et al., The Way of Macroplastic through the Environment. Environments, 2020. 7(10): p. 553

73.

31. Barboza, L.G.A., et al., Chapter 17 - Macroplastics Pollution in the Marine Environment, in World Seas: 555 an Environmental Evaluation (Second Edition), C. Sheppard, Editor. 2019, Academic Press. p. 305-328. 556

32. van Emmerik, T. and A. Schwarz, Plastic debris in rivers. WIREs Water, 2020. 7(1): p. e1398. 557

33. Schwarz, A.E., et al., Sources, transport, and accumulation of different types of plastic litter in aquatic 558 environments: A review study. Marine Pollution Bulletin, 2019. 143: p. 92-100. 559

34. van Emmerik, T., et al., A Methodology to Characterize Riverine Macroplastic Emission Into the Ocean. 560 Frontiers in Marine Science, 2018. 5(372). 561

35. Li, W.C., H.F. Tse, and L. Fok, Plastic waste in the marine environment: A review of sources, occurrence 562 and effects. Science of The Total Environment, 2016. 566-567: p. 333-349. 563

36. Zhao, X., et al., Evaluation of Near-infrared hyperspectral imaging for detection of peanut and walnut 564 powders in whole wheat flour. Applied Sciences, 2018. 8(7): p. 1076.

37. Balakrishnama, S. and A. Ganapathiraju, Linear discriminant analysis-a brief tutorial. Institute for Signal 566 and information Processing, 1998. 18(1998): p. 1-8. 567

38. Fisher, R.A., The use of multiple measurements in taxonomic problems. Annals of eugenics, 1936. 7(2): 568

p. 179-188. 569

39. Kim, S.-J., A. Magnani, and S. Boyd. Robust fisher discriminant analysis. in Advances in neural 570 information processing systems. 2006.

40. Garaba, S.P., et al., Concentration, anisotropic and apparent colour effects on optical reflectance 572 properties of virgin and ocean-harvested plastics. Journal of Hazardous Materials, 2020: p. 124290.

41. Moroni, M., et al., PET and PVC Separation with Hyperspectral Imagery. Sensors, 2015. 15(1): p. 2205- 574 2227.

42. Bonifazi, G., G. Capobianco, and S. Serranti, A hierarchical classification approach for recognition of 576 low-density (LDPE) and high-density polyethylene (HDPE) in mixed plastic waste based on short-wave infrared 577 (SWIR) hyperspectral imaging. Spectrochimica Acta Part A: Molecular and Biomolecular Spectroscopy, 2018.578 198: p. 115-122. 
43. Meacham-Hensold, K., et al., High-throughput field phenotyping using hyperspectral reflectance and

partial least squares regression (PLSR) reveals genetic modifications to photosynthetic capacity. Remote 581 Sensing of Environment, 2019. 231: p. 111176.

44. Danson, F., et al., High-spectral resolution data for determining leaf water content. International Journal of Remote Sensing, 1992. 13(3): p. 461-470.

45. Piarulli, S., et al., Rapid and direct detection of small microplastics in aquatic samples by a new near 585 infrared hyperspectral imaging (NIR-HSI) method. Chemosphere, 2020. 260: p. 127655.

46. Eldin, A. and I. Akyar, Near infra red spectroscopy. Wide spectra of quality control. InTech, Rijeka,

47. Richter, R., et al., Correction of cirrus effects in Sentinel-2 type of imagery. International journal of remote sensing, 2011. 32(10): p. 2931-2941.

48. Guo, X. and P. Li, Mapping plastic materials in an urban area: Development of the normalized difference plastic index using WorldView-3 superspectral data. ISPRS Journal of Photogrammetry and Remote Sensing, 2020. 169: p. 214-226.

49. Fu, B. and I. Burgher, Riparian vegetation NDVI dynamics and its relationship with climate, surface 594 water and groundwater. Journal of Arid Environments, 2015. 113: p. 59-68.

50. Balsi, M., et al., High-Resolution Aerial Detection of Marine Plastic Litter by Hyperspectral Sensing. 596 Remote Sensing, 2021. 13(8): p. 1557.

51. Bonifazi, G., et al., Hyperspectral imaging applied to the waste recycling sector. Spectroscopy europe, 598 2019. 3(2).

52. Pieszczek, L. and M. Daszykowski, Improvement of recyclable plastic waste detection - A novel 600 strategy for the construction of rigorous classifiers based on the hyperspectral images. Chemometrics and 601 Intelligent Laboratory Systems, 2019. 187: p. 28-40. 